\title{
Un musée imaginaire des mondes romani et voyageurs en France
}

Ilsen About, chargé de recherche au CNRS, rattaché à l'IRIS de l'École des hautes études en sciences sociales (EHESS)

Aucun musée n'aborde l'histoire des Roms et des Voyageurs en France dans le temps long et la diversité de leur présence. Aussi, l'ancienneté des mondes romani et voyageurs et leur présence multiple restent peu connues et ne sont que très rarement montrées, plusieurs propositions muséales dans ce sens étant restées sans lendemain, ou presque. Imaginer un musée consacré à l'histoire de ces populations engage le croisement de multiples sources et d'œuvres artistiques qui témoignent des circulations et des ancrages comme des stéréotypes qui sont restés accolés durablement à ces populations.

Imaginer les conditions d'un musée qui exposerait l'histoire des mondes romani et voyageurs en France invite à montrer ce qui disparaît et se révèle derrière les préjugés mais aussi à façonner les formes iconographiques et muséales d'un double impensé. Le premier tient dans l'oblitération collective d'une présence diversifiée et sédimentée dans l'espace français depuis des siècles. Les traits sociaux et culturels des mondes roms, sinti, manouches ou gitans se sont transformés à chaque époque, en s'entremêlant étroitement à l'histoire des territoires du pays. Mais cette présence est demeurée confinée aux marges du récit national et reste largement perçue comme marginale, étrangère, irrégulière et provisoire. Les traces locales et les témoignages sont cependant innombrables et s'inscrivent dans le temps long de l'histoire : elles prennent sens dans tous les contextes des cadres sociaux de la ruralité, de l'urbanité et de l'économie, elles sont circonscrite aux espaces régionaux ou ouverte aux aires frontalières et au monde entier, elles participent d'innombrables domaines de la culture, des arts et des expressions écrites, visuelles ou musicales. Malgré l'existence des témoignages d'une présence à la fois continue et irradiante, la reconnaissance d'une histoire romané de France reste absente presque totalement, et cette absence se traduit par les silences des musées : des œuvres signalent par occurrence les sujets de cette histoire, mais soulignent rarement la nature et l'ancienneté des ancrages auxquels elles se réfèrent. L'existence de documents relatifs aux présences gitanes dans le parcours permanent d'un musée comme celui du Museon Arlaten d'Arles relève ainsi de l'exception.

Le second impensé tient dans la possibilité même d'un musée des mondes romani et voyageurs et sur ce qu'un tel musée pourrait contenir et présenter. Les limites et le périmètre d'un tel lieu posent des questions ouvertes qui n'ont jamais fait l'objet de réels consensus. La France se situe, en effet, à l'intersection de nombreux espaces romani qui se croisent 
rarement, produisant des documentations et des œuvres graphiques d'une extrême hétérogénéité. Les installations à la fin du Moyen Âge, décrites par de nombreux témoignages, et figurées notamment par des tapisseries de Tournai du début du XVI ${ }^{\mathrm{e}}$ siècle laissent supposer des passages plus anciens le long des grands axes fluviaux de l'Europe pontique, méditerranéenne ou atlantique $\mathrm{e}^{\mathrm{i}}$. À ces implantations très anciennes, se sont ajoutées les installations gitanes dans l'espace pyrénéen et dans tout le sud de la France, toutes les diverses composantes des mondes sinti, ou manouches, issus de l'Europe germanique ou de l'Italie, ainsi que les établissements de multiples composantes roms issues de l'Europe du Nord, de l'Est, des Balkans, d'Asie mineure, de Russie et du Caucase. Mais d'autres populations viennent s'ajouter à ces composantes bien étudiées : les Bohémiens d'Ancien régime expulsés vers les îles françaises des Antilles, les multiples groupes qui circulent couramment entre la France et les Nouveaux Mondes, jusqu'au $\mathrm{XX}^{\mathrm{e}}$ siècle, qu'il s'agisse de personnes qualifiées, selon les cas, de Bohémiens du Pérou, de Roms de New York ou de Sinti d'Afrique du Sud ; les Gitans, Roms, Sinti ou Manouches urbanisés de toutes les villes du pays ; les Yéniches de l'Est, les forains, les circassiens, les marchands ambulants et les travailleurs saisonniers ou itinérants, considérés comme des «Forains », des « Marchands ambulants » ou des « Nomades », selon les termes de la loi du 16 juillet 1912 devenue, par la contrainte ou le choix, un creuset de destins communs. Les particularismes locaux et linguistiques, les épreuves de l'histoire, les enclavements, les exils intérieurs forcés par la politique ou les conjonctures personnelles ont aussi créé, répété ou inventé des positions singulières dans l'espace national.

Le musée de ces mondes serait dès lors le produit de cette histoire longue, située dans une multitude de territoires et de trajectoires, et la somme de tous les témoignages disponibles : ceux produit par l'intérêt et la curiosité des observateurs, artistes et savants ainsi que ceux produits par les institutions, notamment celles de l'État, qui ont façonné les cadres d'une intolérance politique et administrative, dominée à l'époque contemporaine par la loi de 1912, la persécution durant la Seconde Guerre mondiale et la poursuite de politiques discriminatoires jusqu'à l'abrogation de la loi de 1969, en 2017 ${ }^{\text {ii }}$. Surtout, il réunirait les témoignages particulièrement méconnus et les expressions des acteurs de ces mondes : simples sujets aux destins conventionnels ou extraordinaires, acteurs des économies pratiques et quotidiennes, artisanales, agricoles ou culturelles, artistes de discrète ou de grande renommée, auteurs et rédacteurs, sous toutes les formes, de leurs langues, de leurs mythologies et de leurs contes, de leurs images et de leurs objets du quotidien, fabricants de leur histoire de France. 


\section{"Un musée de tout ce qui touche des Tsiganes" (André Malraux)}

La grande diversité des mondes romani en France peut expliquer l'absence d'un consensus et d'un projet partagé en vue d'en montrer l'histoire sous forme de musée ou d'exposition. Mais l'histoire contemporaine des porte-paroles et des relais intellectuels ou associatifs et les distances et dissensions qui se sont manifestées semblent aussi avoir découragé toute initiative.

Dès 1956, le comité d'honneur de l'association Études tsiganes, adossée à la revue du même nom, comptait à la fois Paul Rivet, directeur honoraire du musée de l'Homme et Georges Henri Rivière, conservateur du musée national des Arts et Traditions populaires (MNATP), où se sont tenues les permanences régulières de l'association ${ }^{i i i}$. Ces liens structurels se prolongeaient dans l'administration des documents collectés, propriétés de l'association mais conservés par le MNATP : objets, peintures, dessins, estampes, affiches, photographies, phonogrammes furent ainsi déposés pendant des décennies, sous la responsabilité d'un documentaliste de l'association, initialement Philippe Mironneau, et des conservateurs du musée. En 1964, André Malraux, alors ministre des Affaires culturelles, écrivait au président de l'association : il soulignait l'urgence de recueillir tous documents sur les «Tsiganes, Gitans et autres populations d'origine nomade analogue » et insistait sur la nécessité de constituer des données objectives pour contrer les légendes fantaisistes et les préjugés hostiles $^{\mathrm{iv}}$ : «Les Études tsiganes ont commencé à rassembler une documentation qui sera bientôt abritée dans les nouveaux locaux du musée des Arts et Traditions populaires. Il y aurait intérêt à ce qu'elle soit augmentée grâce au dépôt, à titre de don, de prêt ou de simple communication, des documents les plus divers: livres, manuscrits, objets, dessins, photographies, bandes magnétiques notamment. Il faut que naisse un véritable musée de tout ce qui touche des Tsiganes et les populations analogues ${ }^{\nu}$. »

Cet appel surprenant provient du principal architecte des politiques culturelles en France et témoigne d'un intérêt personnel, sans doute guidé par l'ambition d'amplifier l'action et le périmètre du MNATP. En 1966, la recherche au sein du musée devient institutionnelle, par la signature d'une convention entre le Centre national de la recherche scientifique (CNRS) et la direction des Musées de France, et par la création du laboratoire associé, le Centre d'ethnologie française ${ }^{\mathrm{vi}}$. Cette amplification des missions accompagne le projet de réinstallation du MNATP dans le nouveau bâtiment du Bois de Boulogne, inauguré en 1972. Au fil des années, une ample documentation consacrée aux «Tsiganes » est constituée et complétée au gré des collectes et des donations générées par les membres de 
l'association. Si aucun projet de musée ne voit cependant le jour, l'idée d'une exposition est discutée en 1973 et celle-ci devait être organisée au MNATP ${ }^{\text {vii }}$.

Aucun projet d'ampleur ne voit cependant le jour en France, en raison de multiples facteurs, mais peut-être surtout en raison des distances entre les collectifs de Voyageurs et les associations ou les acteurs censés les représenter. Le regard porté sur les prises de parole, les manifestations d'intérêt ou la publication de travaux issus de la recherche est resté longtemps critique alors que des législations discriminatoires restaient en vigueur et que les conditions de vie des Voyageurs, dans de nombreux territoires, se dégradaient au fil du temps. Cet écart et ces tensions apparaissent notamment à l'occasion de la sortie du livre de Marie-Paul Dollé en 1980 sur les Manouches d'Alsace et qui suscite cette réflexion amère de la part d'un homme d'église, le Frère Dominique Joly ${ }^{\text {viii }}$ : « Si le rôle de l'ethnologue consiste seulement à accumuler et à divulguer des connaissances sur les peuples différents de nous, il ne fait que satisfaire le désir d'exotisme de notre société: les Tsiganes se trouvent alors réduits à des objets de consommation. Qu'ils vivent ou non, cela importe peu, puisqu'ils ont leur place au musée de l'Homme $e^{i x}$. »

Ce reproche s'adresse aux chercheurs dont les travaux reproduiraient des visions caricaturales et sans nuance, mais aussi aux collecteurs et responsables d'exposition qui favoriseraient la muséification des sujets et qui oublieraient de les considérer comme des acteurs vivants de la société. À cette époque, plusieurs expositions ou festivals, conçus avec les meilleures intentions, pouvaient paraître ainsi exhausser une différence alimentée par des œuvres artistiques et des programmations qui recouraient à des figurations traditionnelles et stéréotypées $^{\mathrm{x}}$. Ces critiques et les divisions au sein des mouvements associatifs découragent tout projet d'exposition et l'idée d'un musée est abandonnée définitivement. De nouvelles pistes sont ouvertes dans les années 1980, notamment avec la création de l'association Initiatives tziganes, en 1983 et l'organisation, en 1985, d'une Première mondiale d'art tzigane, fondée sur l'idée que l'exposition d'un art tsigane serait en mesure de combattre les préjugés, comme l'explique alors Gérard Gartner: «Le public va voir que nous sommes identiques aux autres hommes, que nous possédons et avons toujours possédé les mêmes valeurs intrinsèques, valeurs humaines et valeurs artistiques. Peut-être plus que certaines manifestations sociales, ce salon nous apportera plus de respects et contribuera à modifier certaines attitudes de rejet à notre égard ${ }^{\mathrm{xi}}$. »

En mai 1985 cette exposition, conçue comme une manifestation symbolique et démonstrative, présente, dans les espaces de la Conciergerie à Paris, les œuvres picturales et plastiques de 35 artistes français et étrangers dont Serge Poliakoff, Otto Mueller, Constantin 
Nepo, Django Reinhardt, Torino Ziegler, Rosa Taikon, Joseph Doerr, Sandra Jayat et Gérard Gartner $^{\mathrm{xii}}$. Les caractéristiques de cette exposition et son caractère novateur résonnent encore de nos jours et ont été notamment invoqués par les inspirateurs du European Roma Institute for Art and Culture, créé en 2017 à Berlin ${ }^{\text {xiii }}$.

L'idéal d'une reconnaissance à travers les expressions artistiques et le regard subjectif des artistes revendiqués en tant que Roms, Sinti ou Gitans a cependant éloigné, en France, tout projet d'un musée qui aurait pour objet l'histoire et les sociétés des mondes romani, en dehors d'initiatives extrêmement localisées et souvent provisoires ${ }^{\mathrm{xiv}}$. En témoigne, la création en 1989 du musée intitulé «Éco-musée tsigane. Panorama du Voyage », situé à quelques kilomètres des Saintes-Maries-de-la-Mer. Ce musée fut créé à l'initiative privée de l'Association saintoise des Gitans et de leurs amis et des efforts coordonnés par Thérèse Chevalier qui a raconté la fabrique de ce lieu : des documents d'histoire, des fresques peintes et des panneaux explicatifs voisinaient avec des roulottes installées sur un terrain où le thé était servi aux visiteurs et spectateurs ${ }^{\mathrm{xv}}$. Cette expérience demeure cependant éphémère et ce musée a disparu à une date inconnue. Au début des années 2000, un Musée des roulottes est créé par un collectionneur, Pierre Le Fur, à Saint Quentin-la-Poterie (Gard) qui insiste sur l'importance de préserver un patrimoine culturel et humain en perdition ${ }^{\mathrm{xvi}}$. En 2013, un projet de Maison de la culture tsigane et gens du voyage de France voit le jour, sous l'impulsion de Monique Riccardi-Cubitt, mais sans donner de suite ${ }^{\mathrm{xvii}}$. Il reste dès lors à imaginer ce que pourrait être un musée des mondes romani et voyageurs qui puiserait à la fois dans tous les arts et dans toutes les œuvres d'artistes disponibles afin de montrer et d'exprimer l'entrelacement d'une population avec l'histoire et la société du territoire de France.

\section{Entrelacements géographiques}

Un premier kaléidoscope visuel devrait rendre compte des innombrables traces topographiques laissées par ces présences : toutes les formes toponymiques possibles, allées, chemins, rues, grottes, lieux-dits dits «des Bohémiens » ou des «Gitans », traduisent les empreintes les plus anciennes, repérées par François de Vaux de Foletier ${ }^{x v i i i}$. Des panneaux dispersés sur les routes d'aujourd'hui rendent encore compte d'une présence disséminée et d'un espace traversé de part en part. Suivre les sentiers tracés par la toponymie serait ainsi le prétexte pour relier les chemins croisés par des observateurs curieux d'époques reculées. On pourrait ainsi repérer les traces laissées par Ulysse Barberen qui croisa la route de Péthiry et sa troupe dans la forêt d'Arithy, dans le Pays basque, au début des années 1850 ; retrouver le lieu d'un enterrement de Bohémiens dans la forêt de Fontainebleau en 1855 ; suivre les traces 
rapportées par Louis Gilbert dans ses visites du pays de Bitche, en Moselle, dans les années 1900 ; parcourir le Morbihan à la suite des Bohémiens dont les présences sont relevées par un observateur au milieu des années $1930^{\text {xix }}$.

Des traces sont aussi restées sur certains murs comme ceux du château de Lourmarin où des «graffiti gitans » énigmatiques, datés du $\mathrm{XVI}^{\mathrm{e}}$ siècle, participent des croyances diffuses mais aussi des proximités à la fois imaginaires et bien réelles entre les Gitans et les populations locales depuis des siècles $^{\mathrm{xx}}$. La Chapelle de Saint-Pierre, une chapelle de pêcheurs à Villefranche-sur-Mer, dans les Alpes-Maritimes, est ornée de fresques de Jean Cocteau (1889-1963) inaugurées en 1957. Le photographe Lucien Clergue (1934-2014) raconte comment ses propres images inspirèrent Cocteau et révèle l'évocation de personnages précis qu'il avait photographiés : «[...] En hommage aux Gitans de Provence, se trouve dans l'entrée de la chapelle à droite le frère de Manitas de Plata: "Polito" joue de la guitare pour une de ses filles, qui danse devant la roulote $[\ldots]^{x x i}$." L'enchevêtrement des présences romané sur les territoires apparait ainsi à travers l'histoire de personnages bien réels que les sources artistiques entremêlées font surgir au fil des rencontres, mises en scène, en récit ou en images.

Il ne faudrait pas ignorer les traces fragiles et fugaces qui montrent l'ancrage au cœur des villes inscrit depuis des générations. Deux petits dessins du peintre Jules Cesbron-Lavau (1831-1921), inspiré par le romantisme, témoignent ainsi de l'installation à Angers, à la fin du $\mathrm{XIX}^{\mathrm{e}}$ siècle de campements sédimentés dans les friches périphériques mais aussi au cœur de la ville, aux abords du château ${ }^{\mathrm{xxii}}$. Les photographies panoramiques des campements près de la foire commerciale de Lille, réalisées en 1929, semblent faire écho lointainement et à distance à ces dessins d'Angers : non loin de l'église Saint-Sauveur, dans un paysage qui ressemble à celui de la zone parisienne, un vaste espace de friches urbaines est peuplé par les habitants de roulottes, de tentes, de bâtis à la fois provisoires et pérennes, suspendus aux plans d'urbanisme qui transforment la ville dans l'entre-deux-guerres ${ }^{\text {xxiii. }}$.

Un examen approfondi de certaines œuvres permet aussi de constater que les évocations apparemment conventionnelles révèlent des dimensions remarquables. Ainsi le tableau d'Eugène Tourneux, daté de 1851, intitulé «Halte d'une famille de Bohémiens », présente, autour d'un joueur de guitare, un groupe de Voyageurs, trois femmes, trois hommes et trois enfants, un baluchon et un bâton de marche au premier plan, installés face à un paysage vallonné au crépuscule. Un document d'archives indique que le premier titre de cette œuvre mentionnait Un retour de vendanges, en vue de la vente du tableau au Musée de Metz, en $1854^{\text {xiv }}$. Au détour de ce changement de titre, une fonction sociale ancienne et méconnue 
des Bohémiens de France apparaît et rappelle leur contribution en tant que saisonniers réguliers de la récolte et la fabrication du vin. Ce tableau fait ainsi écho à une série de clichés des années 1950 conservées au Musée Casa Pairal de Perpignan : un photographe local saisit, lors des vendanges, la présence de Gitans qui s'amusent devant l'opérateur, déplaçant une large roue, se travestissant ou dansant tout sourire ou posant cigarette à la main ${ }^{\mathrm{xxv}}$.

\section{Des professions inscrites dans le territoire}

Les dimensions professionnelles des inscriptions territoriales ne surgissent pas de manière aléatoire mais constituent souvent le cœur même de projets artistiques. Un album réalisé par Charles-Stanislas L'Éveillé (1772-1833) représente l'un des exemples remarquables d'œuvre conçue pour illustrer une présence présentée comme singulière et dangereuse, mais qui témoigne incidemment d'un ancrage économique et social des Gitans dans le sud-ouest de la France ${ }^{\mathrm{xxv}}$. Ingénieur des Ponts-et-Chaussée, grand voyageur en Méditerranée, auteur de traités sur la construction et spécialiste des canaux et des ponts suspendus, L'Éveillé réalise en 1820 un album de dessins en couleur sur les vues et monuments des Pyrénées-Orientales. Fruit de voyages entre 1815 et 1821 dans la région de Perpignan, cinq aquarelles sont consacrées aux Gitanos : Gitanos en voyage, Gitanos en train de voler et dépecer un cheval, Cuisine de Gitanos, Gitans à l'ouvrage et Danse de Gitanos. Au-delà des appréciations péjoratives, ces vues se transforment en documents de nature indiciaire et montrent la fréquentation de lieux précis, les usages vestimentaires et les objets du quotidien, ainsi que les interactions courantes et les échanges notamment commerciaux avec les populations locales. Des fonctions professionnelles précises apparaissent comme l'exercice de la musique et de la danse en public, le ferrage, la tonte et l'entretien des équidés.

À quelques décennies de distance, le photographe excursionniste Eugène Trutat (18401910) saisit, en juillet 1895, plusieurs portraits du Père Espinasse, un Gitan de Saint-Cyprien (Pyrénées-Orientales), tout près de Perpignan ${ }^{\mathrm{xxvii}}$. L'homme, dont le patronyme est traditionnel des Gitans Catalans du Sud-Ouest, arbore une grande moustache, un gilet et une chemise blanche à motif : assis sur un tabouret, les mains sur les genoux, il porte sur l'épaule une large sacoche en cuir contenant tous les outils de la tonte et de l'entretien des chevaux, des mulets et des chiens, une spécialité professionnelle préservée sur la longue durée.

Les arrivées, les passages et les résidences des roms spécialisés dans les métiers de la chaudronnerie, de la vente de chevaux ou du spectacle des ours, autres professions marquantes des mondes romani en France, provoquèrent un grand nombre d'œuvres, photographies, peintures et dessins parfois entrelacés, inscrits dans des espaces précis du 
territoire. Les esquisses préparatoires et le tableau de Diodore Rahoult (1819-1874), Campement de Bohémiens, exposé au Salon de 1868, résonne ainsi avec les photographies prises alors à Grenoble, les vues générales et les portraits, conservées dans les archives de

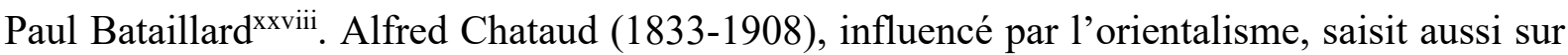
le vif un campement de Bohémiens près d'Alger en 1856, révélant ainsi un espace de circulation élargi à tous les espaces des empires de la France coloniale ${ }^{\mathrm{xxix}}$. À Carcassonne, en 1895, le passage de près de 400 personnes dénommés sous l'appellation de «Tziganes hongrois» laisse alors l'impression d'une «véritable invasion». L'installation du campement, au pied des murailles, donne lieu à un dessin du célèbre illustrateur Henri Meyer (1841-1899) : un groupe d'hommes et de femmes font face aux spectateurs, entourés d'une foule de roulottes de toutes les couleurs, de dizaines de chevaux, d'ours et d'un singe ${ }^{\mathrm{xxx}}$. Ces figurations de «campement de bohémiens » peuvent bien sûr être vues comme des tentatives d'objectivations scientifiques et de dénonciation d'une population mise à distance et enfermée dans les mailles d'un imaginaire canonique. Mais elles sont aussi les traces qui permettent un repérage et la reconstitution d'itinéraires, de déploiements et de circulations au long cours, ainsi que des résidences qui forment autant d'ancrages durables ${ }^{\mathrm{xxxi}}$.

Ce musée ne saurait cependant se limiter à l'exposition des inscriptions de populations dans l'histoire et les territoires. La fabrique politique d'une discrimination inscrite dans la loi et ses conséquences sur la vie des personnes risque de constituer un prisme limité s'il n'est pas articulé au déploiement de multiples parcours de vie. Chacune des trajectoires présentées témoignerait des marques douloureuses de l'histoire mais aussi des combinaisons infinies de la résistance, de l'émancipation et des destinées ordinaires.

\section{Musée du refus et des trajectoires de vie}

André Barthélémy (1914-1991), aumônier des Gens du voyage dans l'après-guerre, avait imaginé qu'un «musée du refus » se composerait idéalement des signes matériels de l'intolérance répartis sur les routes et dans les villages de France, sous forme de panneaux, de pancartes, de plaques parfois scellées sur les murs indiquant «Interdit aux nomades ${ }^{\mathrm{xxxii}} »$. Dans la commune de Bouilly (Aube), une plaque dite de cocher en fonte fixée sur un mur au bord de la route principale est plus explicite encore et indique, en majuscules, le rejet d'une population toute entière : "Défense aux familles nomades de stationner sur le territoire de Bouilly ${ }^{\text {xxiii }}$. $"$ Ces plaques et pancartes, collectées par des collectionneurs, photographiées depuis des décennies, forment une matière étrange et presque contradictoire : l'interdiction ou la limitation du stationnement indique en creux la multitude des espaces de vie habités par 
une population historiquement déplacée, cloisonnée, enfermée et enclavée. Le recensement récent des lieux de vie des Gens du voyage, par William Acker, souligne aussi le remplacement des panneaux d'interdiction par des panneaux de direction vers les «Aires d'accueil des Gens du voyage », bien souvent associées à d'autres directions comme la «Déchetterie », les zones industrielles, les usines, les installations électriques et autres lieux habituellement disjoints des lieux d'habitation ${ }^{\text {xxxiv }}$.

Ces marques infamantes sur le territoire rejoignent d'autres marques apposées sur les corps et les visages depuis l'invention de l'identification moderne : rôles et registres des prisons ou des galères, avis de recherche, relevés de signalement et d'innombrables documents judiciaires de l'Ancien régime témoignent des origines d'une désignation et d'un traitement spécifiques. Le risque d'une interprétation globale et d'une vision uniformisée de la répression peut mener cependant à de nombreux contresens et une exposition critique de l'histoire de l'identification peut permettre de dissocier les périodes et de relever, avec circonspection, que les formulaires de papier sont aussi les dépôts de ce qui est saisi sur le corps même des sujets. Les murs d'images signalétiques de l'identification judiciaire, comme celles conservées miraculeusement des premières campagnes opérées par les Brigades mobiles dans les années 1907-1908, montrent l'intensité et la portée de ces saisies $^{\mathrm{xxxv}}$ : des visages uniformisés par le protocole de l'identification survivent au projet d'encadrement massif d'une population civile et de leur effacement programmé. Des noms et des visages demeurent ainsi que des vêtements, des accessoires, des attitudes contraintes, soumises ou résistantes, et opposées au protocole qui leur est imposé. Des sourires désarment la mécanique du pouvoir, des flocons de neige font irruption et balaient la logique apparemment rigoureuse de l'identification systématique. Pour faire face à l'iconographie policière et aux figurations de l'enfermement et de la répression durant la Seconde Guerre mondiale, sources de très nombreuses images, dessins et peintures, le retournement de perspective peut prendre plusieurs formes et parcourir les innombrables solutions des trajectoires de vie.

À partir des années 1960, des artistes se situent à distance de toutes formes d'objectivation et choisissent de s'immerger au plus près des sociétés romané en France. La démarche de Jean Schmidt (1929-2009), réalisateur du film Kriss Romani sorti en 1963, montre l'ambition mais aussi les limites de cette position : il accumule de très nombreux matériaux, écrits et photographiques, qui témoignent de sa rencontre avec des Roms, Manouches et Gitans de la région parisienne, mais l'histoire du film schématise à l'extrême une supposée mythologie commune et le résultat, contraint par la production, est jugé avec amertume par le réalisateur lui-même ${ }^{\mathrm{xxxvi}}$. Des projets picturaux ou photographiques 
paraissent alors plus à même de montrer la singularité des individus et de souligner une dignité déniée par l'histoire et la société.

Édouard Planchais (1909-1995) présente en 1957 une série de plusieurs dizaines de tableaux d'une série intitulée Les Gitans ${ }^{x x x v i i}$. Il montre la vie en roulottes dans des lieux repérés des Alpes-Maritimes, le long du Var et près de Cagnes-sur-mer mais aussi de nombreux portraits qui égrènent tous les noms des modèles et des habitants des terrains visités par le peintre : Le petit gitan "Quat'sous », Marius, Boba, Paco, La Gitane "La Pilar », Nouni, Balitcho, Carmen Maye, Bernadette, Pola, mais aussi Nouni, Anita et Angela et Le petit Gitan Fernando ${ }^{\mathrm{xx} x \mathrm{iii}}$. Le photographe Alexandre Lewkowicz, né en 1941 en Russie, se forme aux États-Unis et s'installe en France à la fin des années 1960. Il réalise une série intitulée Gitans qui rend compte de sa rencontre, entre 1966 et 1971, avec des Roms originaires des Balkans et installés dans le bidonville de la Courneuve ${ }^{\mathrm{xxxix}}$. Cette série est centrée sur Madeleine Sancho, entourée de sa famille et de ses proches comme Alexandre et Maja Iovanovitch, dans les différentes activités de la vie quotidienne et lors des performances du cirque ambulant qui les relie tous. Cette série rejoint le travail contemporain du peintre d'origine suisse, Jürg Kreienbühl (1932-2007) qui rend compte des conditions de vie dans les bidonvilles de la banlieue parisienne de la fin des années 1950 aux années $1960^{\mathrm{xl}}$. Jeune peintre venu de Bâle à Paris, il s'installe dans un vieil autobus, échoué sur le bidonville de Bezons près de Nanterre et Argenteuil, et partage le quotidien des habitants des lieux et des Gitans qui vivent avec lui. Avec un remarquable sens du détail, Kreienbühl saisit les conditions de vie et les visages, montrant d'un même geste leurs imperfections et leurs qualités dans plusieurs œuvres: Les Nomades de Roumanie (1964), Le vieux manouche “Toussi” (1966) ou Cacan le gitan (l'infirme) (1967).

À la même époque, l'écrivain Matéo Maximoff (1917-1999), à la fois rom par son père et manouche par sa mère, développe une pratique courante de la photographie et du film ${ }^{x l i}$. Muni constamment d'une caméra, il saisit dans des films et photographies, le quotidien des mondes roms, manouches et gitans de toute la France et de la région parisienne où il réside, et tout spécialement autour de Montreuil-sous-Bois. Un album photographique de 51 pages forme une sélection personnelle de photographies dont les plus anciennes remontent au début du $\mathrm{XX}^{\mathrm{e}}$ siècle : à la fois traversée de l'Europe des mondes romani et album de famille, cet ensemble compose un kaléidoscope d'images qui mettent à distance tous les stéréotypes et rapportent toute figuration à l'échelle d'une seule personne, Matéo lui-même, témoin de son histoire, mais aussi collecteur, metteur en scène de la vie de sa famille et de son intimité dont les repères n'appartiennent qu'à lui. Donner à voir cette intimité des personnes et des familles 
constituent à ce titre un enjeu qui questionne la manière de voir une population dans son entier: en 1994, l'écrivain Guy Pierre Geneuil et le photographe Jean-Pierre Bernard consacrent un ouvrage aux Roms de Provence, mais le livre semble finalement rendre compte d'un seul collectif autour de la famille du patriarche Nono Bouchon, qui fut victime des persécutions durant la Seconde Guerre mondiale; le travail sur plusieurs décennies du photographe Mathieu Pernot, en compagnie des membres de la famille Gorgan, porte aussi à ses limites la figuration d'un collectif qui échappe à tout qualificatif, à toute forme d'essentialisation, et se déploie dans l'histoire de chacun des membres d'une même famille ; de même, les albums photographiques de Gilda Demestre, dont elle discute avec l'anthropologue Lise Foisneau, semblent indéchiffrables sans l'histoire intime qui la relie à ces images du quotidien, du temps qui passe et des souvenirs d'une vie ${ }^{\text {xlii. }}$

\section{Conclusion}

Un musée imaginaire des mondes romani et voyageurs en France se construit ainsi à toutes les extrémités d'un spectre qui passe par tous les regards, savants, curieux, policiers, ethnographiques, artistiques. On y trouverait à la fois les quelques images fugaces de l'explorateur et photographe Jean Binot (1867-1909), microbiologiste à l'Institut Pasteur, qui saisit la présence de vanniers au travail près de Chamonix durant l'été 1900 ou des montreurs d'ours qui passent sur la Croisette, à Cannes, en avril 1906 $6^{\text {xliii }}$. On y trouverait aussi un trio en couleur de «Tziganes qui aiment l'air » saisis sur un pont de la Dordogne en 1916 par le photographe Georges Chevalier, un des opérateurs des Archives de la Planète envoyés par le philanthrope Albert Kahn ${ }^{\text {xliv }}$. Comme seraient enfin exposées les œuvres d'artistes, aujourd'hui à la fois méconnus et dont les pièces sont dispersées parfois irrémédiablement : les tableaux et photographies réalisés lorsque Marie Téla Tchaï Winterstein née Vanhootegem (1909-1993) était actrice et modèle dans les années 1930 et les toiles qu'elle réalisa dans l'après-guerre; les tableaux naïfs de Joseph Coucou Doerr (1902-1986), chroniqueur en image et par l'écrit des mondes manouches en France ainsi que les peintures et dessins irréels de Torino Zigler (†2013) qui expose à partir des années 1950 à Paris et en Provence ${ }^{\mathrm{xlv}}$. Ce musée serait ainsi une tentative infinie, peut-être illusoire, pour réparer la discrétion, à la fois forcée et assumée, à laquelle ont été contraintes les expressions culturelles et les présences multiples des mondes romani en Francexlvi.

\footnotetext{
${ }^{\mathrm{i}}$ Denis Bruna, Tsiganes, premiers regards. Craintes et fascination dans la France du Moyen Âge, Lyon, Fage,
} 2014. 
${ }^{\text {ii }}$ La loi no $69-3$ du 3 janvier 1969, relative à l'exercice des activités ambulantes et au régime applicable aux personnes circulant en France sans domicile ni résidence fixe, décidait l'abrogation d'une partie des dispositions de la loi du 16 juillet 1912. Elle comprenait une refonte légère des statuts de marchands ambulants et de forains, remplaçait le carnet anthropométrique imposé aux nomades par un livret de circulation et imposait le rattachement de tout demandeur à une commune dite de rattachement. Voir Marwan Tichani, La difficile libéralisation du régime de contrôle appliqué aux nomades. Genèse de la loi du 3 janvier 1969, mémoire de Master, Rennes, Science Po Rennes, 2012.

iii « Archives et documentation des Études tsiganes », in Études tsiganes, vol. 7, n 3-4, 1961, pp. 23-24.

iv André Malraux, "Lettre du ministre des Affaires culturelles », in Études tsiganes, vol. 10, n 1-2, 1964, pp. 33-34.

${ }^{\mathrm{V}}$ Ibid.

vi Voir Collectif, «Le Musée national des Arts et Traditions populaires et le Centre d'ethnologie française », in L'Homme, vol. 8, n 4, 1968, pp. 125-127 ; Martine Segalen, Vie d'un musée, 1937-2005, Paris, Stock, 2005.

vii « Vie de l'association. Séance du 12 décembre 1973 », in Études tsiganes, vol. 19, n 4, 1973, pp. 53-56. À la même période, des collectes de documents en lien avec les mondes romani sont aussi réalisées ailleurs en Europe dans de nombreux musées qui s'appuient souvent sur d'anciennes collections. Mentionnons les collections constituées par le Musée Ferenc Erkel de Gyula en Hongrie et par le Museum at the Rothenbaum Cultures and Arts of the World, à Hambourg, anciennement Musée d'ethnologie, qui dispose des collections de Max Haferkorn. Voir Kirsten Martins, «Un collectionneur, Max Haferkorn et sa collection », trad. E. von Krausen, in Études tsiganes, vol. 27, $\mathrm{n}^{\circ} 3,1981$, pp. 37-38.

viii Marie-Paul Dollé, Les Tsiganes Manouches, Sand, M.-P. Dollé, 1980.

ix Frère Dominique Joly, « Lorsque les Sinté contestent l'ethnologue », in Monde gitan, n 55, 1980, pp. 11-13.

× Voir par exemple En Alsace avec les Tziganes. Journées culturelles, organisées par l'Appona, Strasbourg (BasRhin), mai-juin 1981, Les Bohémiens, 150 photographies de Pascal Maitre, organisée par l'Association des Gitans et de leurs amis, Saint-Gaultier (Indre), ca. 1980, Manouches en Berry. Aquarelles de M. Angel Rosset, 1895-1979, Association des Gitans et leurs amis, Chateauroux (Indre), 10-28 janvier 1984; Nuits tziganes, Compagnie musicale régionale tzigane de Grasse, Pezenas (Hérault), 19 juillet 1985, Bibliothèque nationale de France, Cabinet des estampes et photographies, OaMat22, Fonds de François de Vaux de Foletier.

${ }^{x i}$ Gérard Gartner, «Initiatives tziganes : première mondiale d'art tzigane », in Études tsiganes, n 1, 1985, pp. 29-30.

xii Bernard Provot, «La Première Mondiale d'Art tsigane a eu lieu », in Études tsiganes, n 2, 1985, pp. 27-28. Voir Sophie Aude, «Image et langage dans les œuvres d'artistes roms contemporains », in Études tsiganes, $\mathrm{n}^{\circ}$ 36, 2008, pp. 10-37; Tania Magy, "Art Rom. Une recherche de terrain en caravane musée », in L'Ethnographie, $\mathrm{n}^{\circ}$ 2, URL : <https://revues.mshparisnord.fr/ethnographie/index.php?id=438\#ftn15>, 2020. Une deuxième exposition internationale, Mondiale d'art tsigane, est organisée en Hongrie en 1995. L'organisation de ces expositions inspirent l'ouvrage de Gérard Gartner, Les sept plasticiens précurseurs tsiganes. Otto Mueller, Serge Poliakoff, Helios Gomez, Tela Tchaï, Django Reinhardt, Constantin Nepo, Yana Rondolotto, Les Ormeaux-de-Baran, Éd. Marinoël, 2011.

xiii Timea Junghaus, Damian Le Bas, «Europe's roma struggle to reclaim their arts scene », in Open Society Foundations Voices, https://www.opensocietyfoundations.org/voices/europe-s-roma-struggle-reclaim-their-artsscene, 31 juillet 2015 .

xiv Au cours de la même période, quelques initiatives notables ont aussi lieu en Europe. Le musée ethnographique de Tarnow, en Pologne, organise en 1979, une exposition spéciale, Les Tsiganes dans la culture polonaise. Voir Muzeum u Tarnowie (dir.), Gyganie W Kulturze Polskiej, Tarnow, Muzeum u Tarnowie, 1979 ; Baptiste Molin, «L'exposition tsigane de Tarnow en Pologne », in Études tsiganes, vol. 25, n 4, 1979, pp. 30-35. Un musée des cultures romani (Muzeum Romské Kultury) est crée à Brno, en République tchèque, en 1991 et le Gordon Boswell Romany Museum est créé à Spalding au Royaume Uni en 1995. Ces exemples demeurent tout à fait isolés.

xv «Aux Saintes-Maries-de-la-Mer », in Monde gitan, n 76, 1990, p. 17 ; Thérèse Chevalier, « Un "éco-musée tsigane" aux Saintes-Maries-de-la-Mer », in Monde gitan, $\mathrm{n}^{\circ}$ 77, 1990, pp. 17-19.

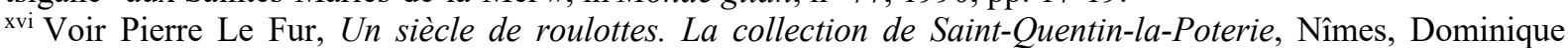
Marck, 2016.

xvii Monique Riccardi-Cubitt, «Roma Cultura. La Maison de la culture tsigane et Gens du voyage de France. Historique », Mediapart, https://blogs.mediapart.fr/monique-riccardi-cubitt/blog/121213/roma-cultura-lamaison-de-la-culture-tsigane-et-gens-du-voyage-de-france, 12 décembre 2013.

xviii François de Vaux de Foletier, «Esquisse d'une toponymie des Tsiganes », in Vie et langage, $\mathrm{n}^{\circ} 192$ et $\mathrm{n}^{\circ} 193,1968$, pp. 148-153 et pp. 224-230.

xix Ulysse Barberen, Une excursion aux Eaux d'Ahunski (Pays basque), Pau, É. Vignancour, 1853 ; Clément Caraguel, "Un enterrement de Bohémiens dans la forêt», in (dir.), Fontainebleau. Paysages, légendes, 
souvenirs, fantaisies, Paris, Hachette, 1855, pp. 197-205; Louis Gilbert, « Les Bohémiens au pays de Bitche », in Le Pays lorrain et le pays messin, 1904, pp. 313-317 ; Étienne Raut, Léon Lallement, " Au sujet des Bohémiens », in Bulletin de la Société polymathique du Morbihan, 1935, pp. 41-52.

xx Sophie Bergaglio, Jules Masson Mourey, «Les graffiti "gitans" du château de Lourmarin (Vaucluse) : mise à l'épreuve d'un morceau de folklore lubéronnais », in Bulletin archéologique de Provence, vol. 40, 2019, pp. 1-9.

xxi Lucien Clergue, " "Le merveilleux échange entre artistes" (Jean Cocteau) », in Revue des deux mondes, 2014, pp. 128-131.

xxii Jules Cesbron-Lavau, Campement de Bohémiens, Pré-Pigeon, Angers et Campement de Bohémiens devant le château, s.d., dessins à la mine de plomb, 20x30, Musée des Beaux-Arts d'Angers.

xxiii Voir l'analyse de Louise Berteloot, «Le recensement du Terrain noir. Réformes urbaines et sociétés de l'itinérance à Lille dans l'entre-deux-guerres », in Genèses [à paraître].

xxiv Eugène Tourneux, Halte d'une famille de Bohémiens, 1851, pastel, 94 x 128 cm., Musée du Louvre. Voir Archives Nationales, F21/58, Recommandation de Prosper Tourneux, frère d'Eugène Tourneux ; le titre « Des Bohémiens » remplace « Un retour de vendanges » sur le dossier, 1849-1854.

${ }^{\mathrm{xxv}}$ Photographe inconnu, Vendanges. Gitans, années 1950, 4 négatifs, 6 x 6 cm, Musée Casa Pairal, Perpignan. Des analyses similaires s'appliquent aussi à bien d'autres œuvres comme celles de Charles Spindler, Alfred Dehodencq ou Mathilde Bonaparte. Voir Sébastien Meyer, «Les Manouches d'Alsace et de Lorraine dans les Arts et les Lettres. Une étude de leur mise à contribution dans la fabrication transrégionale d'une mythologie bohémienne », mémoire de Master, Strasbourg, université de Strasbourg, 2013.

xxvi Cet album est conservé dans les collections des ATP déposées désormais au MUCEM. Voir Olivier Poisson, « L'album inédit de Charles-Stanislas L'Éveillé : "Vues et monumens des Pirénées orientales”, 1820 », in Marie Grau, Olivier Poisson (dir.), Études roussillonnaises offertes à Pierre Ponsich, Perpignan, Le Publicateur, 1987, pp. 377-392 ; Marie-Hélène Solère-Sangla, L'invention d'une Méditerrannée. Patrimoine, création, identité en Roussillon de la fin du XVIII siècle à l'entre-deux-guerres, Perpignan, Presses universitaires de Perpignan, 2016.

xxvii Eugène Trutat, Espinasse père, Gitan de Saint-Cyprien, juillet 1895, plaque de verre, $18 x 13 \mathrm{~cm}$., et Eugène Trutat, Espinasse père, Gitan de Saint-Cyprien, Portrait de face et de profil, juillet 1895, plaque de verre, $15 \times 21$ $\mathrm{cm}$, Muséum de Toulouse.

xxviii Voir Sébastien Meyer, «Exercices de style et regards savants. L'anthropologie des Tsiganes et la photographie au XIX ${ }^{\mathrm{e}}$ siècle », in Ilsen About, Mathieu Pernot, Adèle Sutre (dir.), Mondes tsiganes. Une histoire photographique, 1860-1980, Arles, Actes Sud, 2018, pp. 164-167.

xxix Alfred Chataud, Campement de Bohémiens au champ de manœuvres près d'Alger, 1856, Huile sur carton, 20 x 33 cm., Musée des Beaux-Arts de Pau.

xxx «Une invasion de Hongrois à Carcassonne », in Le Petit journal Supplément illustré, 18 décembre 1898.

xxxi Voir Adèle Sutre, Géopolitique des tsiganes. Des façons d'être au monde, entre circulations et ancrages, Paris, Le Cavalier Bleu, 2021.

xxxii André Barthélémy, «Les terrains de stationnement », in Monde gitan, n 50-51, 1979, pp. 1-4. Voir aussi l'enquête de Jacques Coustals, Luc Romann, La Pancarte, s.1., Jacques Coustals, Luc Romann, 1985

xxxiii Voir la photographie prise par Stéphane Bougel, Défense aux familles nomades de stationner sur le territoire de Bouilly. Url : <https://plaquedecocher.fr/aube-page-5/>

xxxiv William Acker, Où sont les "Gens du voyage” ? Inventaire critique des aires d'accueil, Rennes, Éditions du commun, 2021.

${ }_{\mathrm{xxx}}$ Nicolas Dion, «Nomades ? La numérisation de plaques de verre provenant du fonds photographique du Ministère de l'Intérieur", in Archives nationales, carnet de recherche, URL: $<$ https://labarchiv.hypotheses.org/617>, 2018.

xxxvi Henriette David, « Au-delà de "Kriss Romani”, interview de Jean Schmidt », in Études tsiganes, vol. 9, n 4, 1963, pp. 1-11.

xxxvii Les tableaux d'Édouard Planchais ne semblent pas avoir été conservés au sein d'une collection et n'ont pas pu être localisés.

xxxviii Philippe Mironneau, «L'exposition Édouard Planchais à la galerie Henaut à Paris », in Études tsiganes, vol. $6, n^{\circ} 4,1960$, pp. 21-22.

xxxix Des éléments de cette série figure dans les collections du Mucem et dans celles de la BnF.

${ }^{x l}$ Je remercie Jean Marie Oger de m'avoir fait connaître l'œuvre de Jürg Kreienbühl mise en valeur par la galerie Jean-Marie Oger à Paris.

xli « Matéo Maximoff. Un écrivain rom dans le siècle », Études tsiganes, n 60, 2017. Ses archives sont déposées auprès de la Médiathèque Matéo Maximoff à Paris.

xlii Jean-Pierre Bernard, Guy Pierre Geneuil, Ils portent en eux leur rêve. Les Rom de Provence, Paris, Dervy, 1994 ; Mathieu Pernot, Les Gorgan, 1995-2015, Paris, Xavier Barral, 2017 ; Lise Foisneau, " "Se donner en 
photo". Le purane pildi la Gildake, les vieux clichés de Gilda ", in Hommes \& Migrations, n 1321, 2018, pp. 112-116.

xliii Jean Binot, Bohémiens près de Chamonix, Mer de glace, de la route, 1900, Cannes, Croisette, Bohémiens, 1906 plaques de verre, 4,5x10,7 cm., Musée du Quai Branly.

xliv Georges Chevalier, Près des Eyzies, Tziganes qui aiment l'air, le garçon avec un pantalon espagnol, 1916, Siorac-en-Périgord (Dordogne), 16 juin 1916, Collection du Musée Albert Kahn.

xlv Klaus-Michael Bogdal, «"Ein Zigeunerin ist keine Konkurrenz für Dich!” Der vergessene französische Filmstar Tela Tchaï/Martha Winterstein. Ein Spurensuche », in Marina Ortrud M. Hertrampf, Kirsten von Hagen (dir.), Ästhetik(en) der Roma, München, AVM, 2020, pp. 185-208; Joseph Doerr, Où vas-tu, Manouche ?Vie et mœurs d'un peuple libre, Bordeaux, Éditions Wallada, 1982 ; Jean-Luc Poueyto, « Coucou Doerr, un écrivain naïf? », in Etudes Tsiganes, n $^{\circ} 37,2009$, pp. 118-129; Florence Dalle, «La peinture de Torino Zigler », in Études tsiganes, vol. 14, ${ }^{\circ} 1,1968$, pp. 8-12.

xlvi Voir l'analyse de Jean-Luc Poueyto, « Un patrimoine culturel très discret : le cas des Manouches », Terrain, $\mathrm{n}^{\circ} 58,2012$, pp. 130-143. 